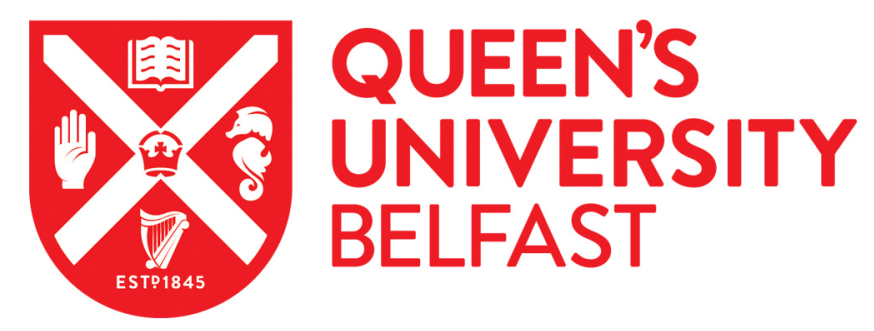

\title{
KRAS Allelic Imbalance Strengths and Weaknesses in Numbers
}

Doherty, G. J., Kerr, E. M., \& Martins, C. P. (2017). KRAS Allelic Imbalance Strengths and Weaknesses in Numbers. Trends in molecular medicine, 23(5), 377-378. https://doi.org/10.1016/j.molmed.2017.03.005

\section{Published in:}

Trends in molecular medicine

\section{Document Version:}

Peer reviewed version

Queen's University Belfast - Research Portal:

Link to publication record in Queen's University Belfast Research Portal

\section{Publisher rights}

Copyright 2017 Elsevier.

This manuscript is distributed under a Creative Commons Attribution-NonCommercial-NoDerivs License

(https://creativecommons.org/licenses/by-nc-nd/4.0/), which permits distribution and reproduction for non-commercial purposes, provided the author and source are cited.

\section{General rights}

Copyright for the publications made accessible via the Queen's University Belfast Research Portal is retained by the author(s) and / or other copyright owners and it is a condition of accessing these publications that users recognise and abide by the legal requirements associated with these rights.

Take down policy

The Research Portal is Queen's institutional repository that provides access to Queen's research output. Every effort has been made to ensure that content in the Research Portal does not infringe any person's rights, or applicable UK laws. If you discover content in the Research Portal that you believe breaches copyright or violates any law, please contact openaccess@qub.ac.uk. 
KRAS Allelic Imbalance: Strengths and Weaknesses in Numbers

Gary J. Doherty ${ }^{1,2}$, Emma M. Kerr ${ }^{1}$, Carla P. Martins ${ }^{1, *}$

${ }^{1}$ MRC Cancer Unit, University of Cambridge, Hutchison/MRC Research Centre, Box 197, Cambridge Biomedical Campus, Cambridge, CB2 0XZ, UK.

${ }^{2}$ Department of Oncology, University of Cambridge, Addenbrooke's Hospital, Box 193, Cambridge Biomedical Campus, Cambridge, CB2 OQQ, UK.

*Corresponding author: c.martins@mrc-cu.cam.ac.uk 


\section{Abstract}

The identification of therapeutic vulnerabilities in mutant KRAS tumors has proven difficult to achieve. Burgess and colleagues recently reported in Cell that mutant/wild-type Kras allelic dosage determines clonal fitness and MEK inhibitor sensitivity in a leukemia model, demonstrating that KRAS allelic imbalance is likely an important and overlooked variable. 
The RAS family of small GTPases controls multiple signaling cascades, including the RAF/MEK/ERK (MAPK) and PI3K/AKT/mTOR pathways. Point mutations in codons G12, G13 and Q61 result in the constitutive activation of the KRAS oncogene and are highly prevalent in epithelial malignancies, namely pancreatic, colorectal and lung cancers [1]. KRAS mutations are also found -- albeit at a lower frequency -- in hematological malignancies, affecting approximately $5 \%$ of acute myeloid leukemias (AML) $[2,3]$. As mutant KRAS itself remains untargetable, the inhibition of its downstream effectors represents an alternative therapeutic strategy for mutant KRAS cancer treatment. However, this approach has had limited success to date [4, 5], reflecting our incomplete understanding of mutant RAS activity in cancer. Burgess and colleagues now show that in murine AML and human colorectal cancer cell lines, mutant/wild-type (WT) KRAS allelic balance dictates sensitivity to MEK inhibition [6].

To identify genetic signatures of sensitivity or resistance to MEK inhibition, the authors generated genetically diverse murine $\mathrm{Kras}^{G 12 D}$-driven $\mathrm{AML}$ in vivo, using retroviral insertional mutagenesis. Four independent primary AMLs were subsequently transplanted into recipient mice and treated with control vehicle, or the MEK1/2 inhibitor PD0325901. While three AMLs showed only a mild improvement in recipient survival following MEK inhibition, one leukemia (AML101) was particularly sensitive to this treatment. However, PD0325901 treatment eventually selected for the emergence of resistant clones within AML101; indeed, cells isolated from treated animals and then subjected to subsequent PD0325901 treatment exhibited poorer treatment responses than AML101 in vitro and in vivo.

Further characterization of the "super PD0325901-responder" AML101 and its derived resistant clone (AML101-R) revealed Kras allelic imbalance in both settings. Using a combination of whole exome sequencing and fluorescence in situ hybridization (FISH), the authors showed that, in contrast to the donor mouse $\left(K_{r a s^{G 12 D /+}}\right)$, AML101 was homozygous for the mutant $K_{r a s^{G 12 D}}$ allele, with this duplication being acquired through uniparental disomy. AML101-R cells also exhibited two mutant alleles, but in this case, they were accompanied by a WT copy (G12D:WT ratio=2:1) (Figure 1). Since in the three other AMLs that were only mildly sensitive to MEK inhibition the 
Kras WT allele was also retained (albeit to variable degrees), retention of WT Kras correlated directly with resistance to MEK inhibition in AML. In agreement, the sensitivity of mutant homozygous AML101 cells to PD0325901 treatment in vitro was reduced upon overexpression of WT Kras.

FISH analysis suggested that the PD0325901 resistant clone was present at low frequency prior to treatment (approximately $3 \%$ ). In competitive in vivo assays carried out in the absence of treatment, AML101-R exhibited decreased fitness relative to AML101 explaining the preferential expansion of AML101 prior to treatment and indicating that loss of WT Kras provides a growth advantage to mutant homozygous cells (Figure 1). A growth inhibitory role for WT Kras was previously reported in carcinogen-induced lung tumors, where despite the presence of carcinogen-induced Kras mutations in all lung lesions, tumor development was significantly accelerated in $\mathrm{Kras}^{+/}$mice relative to WT animals [7]. By contrast, upon MEK inhibition, the relative fitness of the two AML clones was reversed (Figure 1), with WT Kras conferring a growth advantage to AML cells. WT KRAS was also previously shown to provide a benefit to mutant KRAS colorectal cancer cells by reducing mutant-induced apoptosis [8]. Collectively, these findings highlight the context dependence and complexity of the interplay between WT and mutant KRAS activity in tumors.

Despite being significantly enriched in certain cytogenetic subtypes, KRAS mutations are relatively uncommon in AML [2]. These mutations are, as mentioned, a common feature of epithelial cancers [1], prompting Evangelista and Shannon's groups to examine the applicability of their AML findings to human epithelial cancers. Mutant KRAS allelic imbalance is frequently found in human pancreatic, lung and colorectal cancer cell lines, as well as tumor samples of different origin $[6,9]$. The authors showed that colorectal cancer cell lines with high mutant KRAS allelic frequency (mutant:WT allelic ratio>1) were sensitive to MEK inhibition, while the remaining cell lines displayed variable responses [6]. It is unclear how WT Kras modulates sensitivity to MEK inhibition in murine AML and human colorectal cancer cell lines, and whether a similar mechanism may be at play in human tumors that responded poorly to MEK inhibition in clinical trials $[4,10]$. However, since no correlation between KRAS status and PD0325901 treatment 
responses could be found in pancreatic and lung cancer cell lines [6], the therapeutic susceptibilities associated with KRAS allelic imbalance are likely to be context- and/or tissuedependent.

Oncogene dosage is an important emerging concept that takes into account the effect of cancer mutations not only from a qualitative perspective (presence/absence of mutation), but also quantitatively (copy gain/loss). Given the high incidence of chromosome gains/losses in human cancers (see http://cancer.sanger.ac.uk/cosmic), the extent to which oncogene dosage can impact tumor development and therapy may therefore have been underestimated. In the case of $K R A S$, there is now increasing evidence that relative mutant dosage can have a major effect across different tumor types. Indeed, our laboratory recently showed that in murine lung tumor models, mutant copy gain ( $\operatorname{ras}^{G 12 D / G 12 D}$ versus $\mathrm{Kras}^{\mathrm{G12D/+}}$ ) increases the metastatic potential of tumor cells and rewires their glucose metabolism, creating unique metabolic dependencies and therapeutic vulnerabilities. A similar mutant KRAS copy gain dependent metabolic rewiring was observed in human non-small cell lung cancer cells lines [9].

Collectively, these novel findings suggest that the heterogeneity of mutant/WT KRAS allelic content displayed by human cancers can have important therapeutic implications and may have contributed to the poor therapeutic responses often observed in the treatment of mutant KRAS tumors $[4,5]$. Stratification of KRAS mutant tumors according to their KRAS allelic content (presence/absence of WT allele, mutant and WT copy number) might thus aid the identification of therapeutic vulnerabilities within this heterogeneous group of diseases, and may ultimately contribute to improved patient care. 


\section{References}

1. Pylayeva-Gupta, Y. et al. (2011) RAS oncogenes: weaving a tumorigenic web. Nat Rev Cancer $11(11), 761-74$.

2. Papaemmanuil, E. et al. (2016) Genomic Classification and Prognosis in Acute Myeloid Leukemia. New England Journal of Medicine 374 (23), 2209-2221.

3. Ward, A.F. et al. (2012) Targeting oncogenic Ras signaling in hematologic malignancies. Blood 120 (17), 3397-406.

4. Stephen, A.G. et al. (2014) Dragging ras back in the ring. Cancer Cell 25 (3), 272-81.

5. McCormick, F. (2015) KRAS as a Therapeutic Target. Clinical Cancer Research 21 (8), 1797.

6. Burgess, M.R. et al. (2017) KRAS Allelic Imbalance Enhances Fitness and Modulates MAP Kinase Dependence in Cancer. Cell 168 (5), 817-829.e15.

7. Zhang, Z. et al. (2001) Wildtype Kras2 can inhibit lung carcinogenesis in mice. Nat Genet 29 (1), 25-33.

8. Matallanas, D. et al. (2011) Mutant K-Ras activation of the proapoptotic MST2 pathway is antagonized by wild-type K-Ras. Mol Cell 44 (6), 893-906.

9. Kerr, E.M. et al. (2016) Mutant Kras copy number defines metabolic reprogramming and therapeutic susceptibilities. Nature 531 (7592), 110-113.

10. Stinchcombe, T.E. and Johnson, G.L. (2014) MEK inhibition in non-small cell lung cancer. Lung Cancer 86 (2), 121-125. 


\section{Figure 1: Differential Effects of Kras Imbalance in AML.}

Murine AML cells with a Kras ${ }^{G 12 D}$ allele duplication that either retain or lose the WT Kras copy display differential growth capacity both in the presence and absence of the MEK1/2 inhibitor PD0325901. In particular, Kras ${ }^{G 12 D / G 12 D}$ cells exhibit a proliferative advantage in the absence of treatment but are more sensitive to MEK inhibition relative to cells that retain the WT Kras allele [6]. 


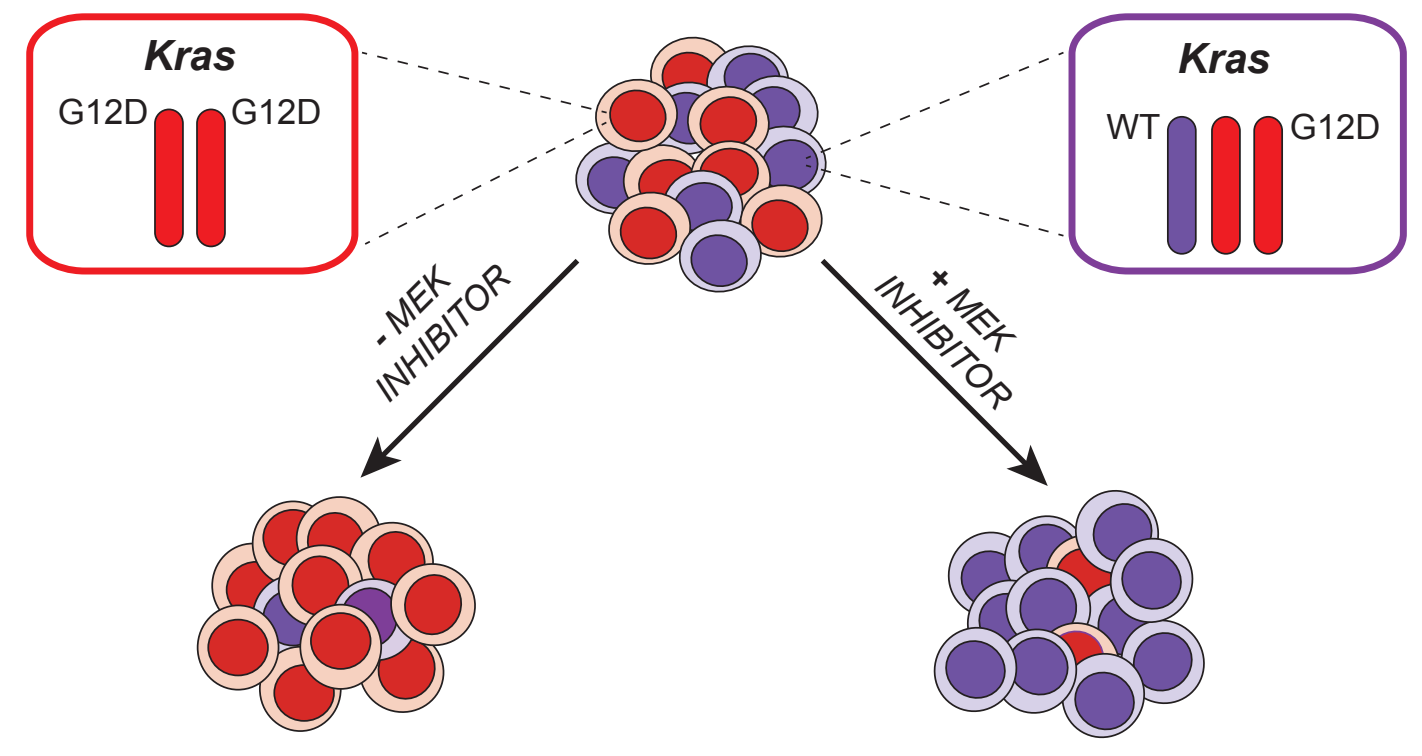

Higher proliferative capacity STRENGTH MEK inhibitor resistance 